\title{
Thrombolyse auch noch sechs Stunden nach dem Schlaganfall
}

Die Thrombolyse bei akutem ischämischem Schlaganfall ist derzeit nur bis 4,5 Stunden nach Symptombeginn zugelassen. Die IST-3-Studie hat nun ergeben, dass die Thrombolyse den Patienten auch noch bis zu sechs Stunden - unabhängig vom Alter und anfänglicher Schwere des Schlaganfalls - nützen kann.

- In die IST-3-Studie wurden $3035 \mathrm{~Pa}$ tienten mit akutem ischämischem Schlaganfall aufgenommen, davon waren 53\% älter als 80 Jahre. Nach der Randomisierung erhielten $1515 \mathrm{~Pa}$ tienten innerhalb von sechs Stunden nach Symptombeginn eine i.v. Thrombolyse mit rekombinantem Tissue Plasminogen Aktivator (rtPA; 0,9 mg/kg Körpergewicht, davon $10 \%$ als Bolus). Die übrigen 1520 Patienten bekamen i.v. Placebo zusätzlich zur spezialisierten Behandlung in einer Stroke Unit. 71\% der Patienten in beiden Gruppen wurden im Zeitraum von drei bis sechs Stunden nach Symptombeginn behandelt. Primärer Endpunkt war das Überleben ohne Alltagsbehinderung (defi- niert als ein Punktwert von $<2 / 5$ auf der Oxford Handicap Skala) nach sechs Monaten.

Die Mortalität war in der ersten Woche höher in der rtPA-Gruppe (11 vs. $7 \%)$, was teilweise durch eine erhöhte Rate intrakranieller Blutungen in der ersten Woche erklärt wird (7 vs. 1\%). Nach sechs Monaten bestand in der Mortalität jedoch kein Unterschied mehr zwischen den beiden Gruppen (jeweils 27\%).

Der Anteil der Patienten, die nach sechs Monaten überlebt hatten und im Alltag unabhängig waren, war in der rtPA-Gruppe mit 37\% leicht, aber statistisch nicht signifikant höher als in der Placebogruppe (35\%, p =0,181). Allerdings hatten Patienten der rtPA-Gruppen nach sechs Monaten signifikant geringere Punktwerte auf der Oxford Handicap Skala (Odds Ratio $=1,27$ ), entsprechend einer um 27\% höheren Chance einer geringeren Behinderung durch die Thrombolyse.

In Untergruppenanalysen profitierten vor allem Patienten über 80 Jahren und solche mit klinisch schweren Schlaganfällen von der Thrombolyse.

\section{Kommentar}

Mit der Thrombolyse steht seit vielen Jahren eine hoch wirksame Therapie zur Verfügung, durch die die Wahrscheinlichkeit eines Überlebens ohne Alltagsbehinderung signifikant erhöht wird, wenn sie rechtzeitig eingesetzt wird. Die IST-3 Studie bestätigt nun erneut die Wirksamkeit der Thrombolysetherapie bei akutem ischämischem Schlaganfall. Darüber hinausgehend zeigen die Ergebnisse jedoch auch, dass wahrscheinlich mehr Patienten von der Thrombolyse profitieren können, als durch die gegenwärtige Zulassung des Medikaments rtPA abgedeckt ist. Dies gilt vor allem für Patienten über $80 \mathrm{Jah}$ ren, solche mit schweren Schlaganfällen und für Patienten, bei denen die Therapie erst im Fenster zwischen 4,5 und sechs Stunden begonnen werden kann. Diese Patientengruppen wurden bislang von der Thrombolyse ausgeschlossen.

M. BusCH =

- The IST-3 collaborative group .

The benefits and harms of intravenous thrombolysis with recombinant tissue plasminogen activator within $6 \mathrm{~h}$ of acute ischaemic stroke (the third international stroke trial [IST-3]): a randomised controlled trial. Lancet 2012; 379: 2352-2363

\section{Achtung:}

\author{
Hier muss der \\ Dummy durch \\ eine Anzeige \\ ersetzt werden !!
}

